\title{
A multi-center randomized controlled trial to reduce unmet needs, depression, and anxiety among hematological cancer patients and their support persons
}

William Stevenson, Jamie Bryant, Rochelle Watson, Rob Sanson-Fisher, Christopher Oldmeadow, Frans Henskens, Christina Brown, Sundra Ramanathan, Campbell Tiley, Anoop Enjeti, Johanna Guest, Flora Tzelepis, Christine Paul \& Catherine D’Este

To cite this article: William Stevenson, Jamie Bryant, Rochelle Watson, Rob Sanson-Fisher, Christopher Oldmeadow, Frans Henskens, Christina Brown, Sundra Ramanathan, Campbell Tiley, Anoop Enjeti, Johanna Guest, Flora Tzelepis, Christine Paul \& Catherine D'Este (2019): A multi-center randomized controlled trial to reduce unmet needs, depression, and anxiety among hematological cancer patients and their support persons, Journal of Psychosocial Oncology, DOI: 10.1080/07347332.2019.1692991

To link to this article: https://doi.org/10.1080/07347332.2019.1692991

2019.

Џ Article views: 30

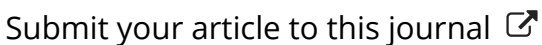

\section{View Crossmark data \lceil}




\title{
A multi-center randomized controlled trial to reduce unmet needs, depression, and anxiety among hematological cancer patients and their support persons
}

William Stevenson, PhD, MBBS ${ }^{\mathrm{a}}$ (D), Jamie Bryant, PhD, BPsyc (Hons) ${ }^{\mathrm{b}, \mathrm{c}, \mathrm{d}}$ (D) Rochelle Watson, BPsyc (Hons) ${ }^{\mathrm{b}, c, d}$ (D), Rob Sanson-Fisher, PhD, M. Psychology (Clin), BPsyc (Hons) $)^{b, c, d}(\mathbb{D})$, Christopher Oldmeadow, PhD, B. Math (Hons) ${ }^{\text {d,e }}$ (ID, Frans Henskens, PhD, Dip. Ed, DCSE, B. Math ${ }^{b, c, d}$ (D), Christina Brown, MBBS, PhD, FRACP, FRCPA ${ }^{f}$ (D), Sundra Ramanathan, MBBS, FRACP, FRCPA ${ }^{g}$ (D), Campbell Tiley, MBBS, FRACP, FRCPA ${ }^{\mathrm{h}}$ (D) Anoop Enjeti, MBBS, MD, FRCP, FRCPA, FASCP, FACP, MClinEpid ${ }^{d, i j, j, k}$ D, Johanna Guest, GradCert. Onc. Nurs., BHS (Nurs), DHS (Nurs) $^{\prime}$ (D) Flora Tzelepis, PhD, BS (Hons) ${ }^{c}$ (D) Christine Paul, PhD, BA (Hons) $^{\mathrm{m}}\left(\mathrm{D}\right.$, and Catherine D'Este, PhD, B. Math, GradDip Med. Stat., Dip. Ed ${ }^{\mathrm{k}, \mathrm{m}}$ (D)

\begin{abstract}
aDepartment of Haematology, Royal North Shore Hospital, Kolling Institute of Medical Research, University of Sydney, St Leonards, New South Wales, Australia; ${ }^{b}$ Faculty of Health and Medicine, Health Behaviour Research Collaborative, School of Medicine and Public Health, University of Newcastle, Callaghan, New South Wales, Australia; 'Priority Research Centre for Health Behaviour, University of Newcastle, Callaghan, New South Wales, Australia; ${ }^{\mathrm{d}}$ Hunter Medical Research Institute, New Lambton Heights, New South Wales, Australia; ${ }^{\mathrm{e} C l i n i c a l}$ Research Design, IT and Statistical Support Unit, Faculty of Health and Medicine, School of Medicine and Public Health, University of Newcastle, Callaghan, New South Wales, Australia; fDepartment of Haematology, Royal Prince Alfred Hospital, University of Sydney, Camperdown, New South Wales, Australia; ${ }^{9}$ Department of Haematology, St George Hospital, Kogarah, New South Wales, Australia; ' Department of Haematology, Gosford Hospital, Gosford, New South Wales, Australia; 'Haematology Unit, Calvary Mater Newcastle, Waratah, New South Wales, Australia; ${ }^{j}$ NSW Health Pathology North-Hunter, New Lambton Heights, New South Wales, Australia; ${ }^{k}$ National Centre for Epidemiology and Population Health, Research School of Population Health, Australian National University, Canberra, Australian Capital Territory, Australia; 'Department of Haematology, Royal North Shore Hospital, St Leonards, New South Wales, Australia; ${ }^{\mathrm{m} S c h o o l}$ of Medicine and Public Health, Faculty of Health and Medicine, University of Newcastle, Callaghan, New South Wales, Australia
\end{abstract}

\begin{abstract}
Purpose: Individuals diagnosed with a high-grade hematological malignancy are at high risk for psychosocial distress. This study aimed to examine the effectiveness of a web-based information tool and nurse delivered telephone support in reducing: (i) unmet information needs; (ii) depression; and (iii) anxiety, among hematological cancer patients and their support persons (SPs).

Methods: Patients with a new diagnosis of acute myeloid leukemia, acute lymphoblastic leukemia, Burkitt lymphoma, or lymphoblastic lymphoma and their SPs were enrolled in a prospective multi-site randomized trial. Participants received
\end{abstract}

\section{KEYWORDS}

Anxiety; depression; hematologic neoplasms; leukemia; randomized controlled trial

CONTACT Jamie Bryant, PhD, BPsyc (Hons) jamie.bryant@newcastle.edu.au E Health Behaviour Research Collaborative, School of Medicine and Public Health, Faculty of Health and Medicine, University of Newcastle, Callaghan, New South Wales 2308, Australia. 
either access to an online information tool and telephone support from a hematology nurse, or usual care. Outcome data were collected 2, 4, 8, and 12 weeks post-recruitment. The primary endpoint was unmet information needs.

Results: Data from 60 patients and 15 SPs were included in the analysis. There were no statistically significant differences in unmet information needs, depression or anxiety between intervention and control groups for patients. Patients in both groups demonstrated a decrease in information needs over the intervention period. Post hoc analyses revealed that patients who did not achieve remission with the first cycle of treatment experienced increased anxiety from 4 weeks until the end of the study $(p=0.008)$.

Conclusions: A web-based information tool and nurse delivered telephone support did not reduce unmet information needs, depression or anxiety among hematological cancer patients, however this finding is inconclusive given the low power of the study.

Implications for Psychosocial Providers or Policy: Patients who do not achieve remission are at high risk of anxiety, and may benefit from targeted psychological intervention.

\section{Introduction}

Individuals diagnosed with a high grade hematological malignancy are likely to experience prolonged hospital admission associated with intensive chemotherapy. ${ }^{1}$ Treatment often commences rapidly following admission, and information is provided when patients are undergoing several painful procedures, commencing a multi-drug chemotherapy protocol, ${ }^{2}$ and facing prolonged absence from work or training and significant mortality and morbidity risks. Newly diagnosed hematological cancer patients experience higher levels of anxiety $(23 \%)^{3}$ and depression $(17 \%)^{3}$ than those with other cancer types. Hematological cancer patients (27\%) and their SPs (SPs; $29 \%$ ) also report unmet supportive care needs in relation to finances, emotional support, and information. ${ }^{4}$ Specific information needs were related to accessing up-to-date information (9\%) presented in a way they could understand $(7 \%){ }^{4}$ It is critical that supportive care interventions are developed to assist these patients to deal with the psychosocial impacts of their cancer diagnosis.

Acute hematological cancer patients often have limited time to make decisions about whether or not to have a specific treatment, such as intensive multi-agent chemotherapy, or palliative care with transfusion support, or be involved in clinical trials. Ensuring patients are provided with tailored information to enable decision making is important for minimizing unmet information needs and distress. ${ }^{5}$ Several interventions have been effective in improving engagement of cancer patients in decision making, decreasing 
anxiety, and improving understanding of cancer-related information. ${ }^{6}$ Such strategies include treatment decision-aids, ${ }^{7}$ patient question prompt lists, ${ }^{6}$ multiple consultations, ${ }^{8}$ and educational videos to prepare patients for what to expect during treatment. ${ }^{9}$ While these strategies have been demonstrated to enhance patients' understanding of cancer related information, there is strong evidence that patients differ in the amount and type of information they wish to receive, and their preferred formats for accessing information. ${ }^{10,11}$ Tailoring information to meet the needs and preferences of patients improves information recall, reduces anxiety, and improves satisfaction with care. ${ }^{12,13}$

The internet is increasingly used by cancer patients as a resource for information and support. ${ }^{14,15}$ Web-based interventions can be structured around the preferences of patients, ${ }^{16}$ allowing them to determine when, what type, and how often they access information. ${ }^{16}$ Tailored web-based information has numerous advantages over standard information that can be located on the internet. Several reviews report evidence for the effectiveness of Web-based interventions for improving psychosocial outcomes; however, the included studies were limited by lack of scientific rigor and mixed findings. ${ }^{17,18}$ These reviews also reveal a paucity of published research examining the effectiveness of Web-based interventions to improve psychosocial outcomes for hematological cancer patients specifically, with previous trials typically focusing on outpatients and patients later in the disease trajectory. There is a need to evaluate the effectiveness of Web-based interventions in this under-researched group.

\section{Aims}

\section{Primary aim}

To determine, using a prospective randomized controlled trial, the effectiveness of a Web-based information tool and nurse delivered telephone support in reducing unmet information needs among hematological cancer patients and their SPs.

\section{Secondary aims}

To determine the effectiveness of the intervention in reducing depression and anxiety among hematological cancer patients and their SPs.

\section{Methods}

Detailed study methodology is published elsewhere. ${ }^{19}$ Ethics approval was provided by the University of Newcastle Human Research Ethics Committee and Hunter New England Human Research Ethics Committee. 
Approval was also provided by the research governance committees of each participating hospital.

\section{Study design}

A prospective non-blinded multi-site randomized controlled trial.

\section{Participants}

Patients were eligible if they had a new diagnosis of acute myeloid leukemia (AML), acute lymphoblastic leukemia (ALL), Burkitt lymphoma, or lymphoblastic lymphoma. This group of cancer patients was selected as it was hypothesized they may have the greatest psychological burden and unmet information needs given the complexity and intensity of their medical treatment that typically requires an initial prolonged admission to hospital of approximately $4-5$ weeks. $^{20}$ To participate, patients were required to be aged 18 years or older; English speaking; potentially making a decision regarding their treatment; and have a life expectancy of $>2$ months as judged by their clinician. Patients were excluded if they had commenced cytotoxic chemotherapy. SPs were eligible to participate if they were aged 18 years or older and were nominated by a consenting patient as an important source of support in relation to their cancer. Patients could still participate if they did not have a SP.

\section{Recruitment}

Patients were recruited from adult hematology inpatient wards within five hospitals in New South Wales, Australia. Recruitment occurred as soon after diagnosis as possible. Eligible patients were provided with information about the study and invited to participate by their doctor. A study information statement, consent form, and reply-paid envelope was provided to consenting patients to pass on to their nominated SP. SPs were instructed to return a signed consent form to the researchers if they wished to participate.

\section{Randomization}

A random allocation sequence was generated using an online random number generator, with weeks allocated as intervention or usual care according to the sequence. For example, if it was an intervention week, all participants recruited during that week were allocated to the intervention group. The clinical trial nurse at each participating hospital was informed at the start of each week of the allocation sequence and was responsible 
for communicating this to the hematologists involved in recruitment at their site. Where a consenting patient had a consenting SP, they were randomized as a dyad. All participants and healthcare providers were blind to allocation sequence; however, due to the nature of the trial, it was not possible to blind participants or healthcare providers to condition allocation. Participants were blind to condition allocation until after they had provided written consent for participation.

\section{Intervention}

\section{Web-based information tool}

The development of the intervention has previously been described. ${ }^{19}$ Briefly, the Web-based tool included information tailored to the circumstances of the patient about: cancer and its causes; treatment options; complementary and alternative medicine; impact of cancer and treatment; available support services; links to relevant websites; and a discussion forum allowing participants to interact. At the time of enrollment into the study, clinicians were able to tailor the information to the specific hematological cancer diagnosis and appropriate treatment options including types of chemotherapy treatments, clinical trials and palliative approaches for the patient. The intervention included information targeted at both patients and SPs. Content was developed using resources from the Leukemia Foundation of Australia, Cancer Council NSW and Macmillan Cancer Support UK, and refined through multi-disciplinary advisory group meetings. The information tool was pilot tested with 33 hematology cancer patients who were asked to rate sections of the website and complete a brief survey about the acceptability of the intervention. Consumer feedback was evaluated and incorporated if recommended changes reflected the views of the majority.

Use of the Web-based information intervention was self-guided, with participants accessing the tool as often or as little as they wanted. The intervention was designed to be accessed at the time of diagnosis and throughout the treatment course. Participant interaction with the site was recorded by the software. Participants could access the tool using their own Internet-enabled device, and touch screen tablet devices were available to participants to facilitate use. At recruitment, participants were provided written instructions about accessing the tool, and received a demonstration of the tool by research nurses and/or research volunteers. This information was repeated at each outcome time point either in person by the research nurse/volunteer or via an information leaflet mail out with the survey. 


\section{Nurse-delivered telephone support}

Participants in the intervention condition were also informed that they had access to an experienced hematological cancer nurse to ask general questions about their diagnosis and treatment. Participants could contact the nurse by either calling a toll-free telephone number, or indicating they would like the nurse to telephone them by clicking an icon within the Web-based tool. The inclusion of nurse-delivered telephone support was intended to: supplement the information included in the web-based tool; address any patients' questions or concerns which emerge following review of the information; and minimize the burden on hospital staff in meeting information needs.

\section{Usual care}

Participants in the usual care condition received the level of care usually provided at their treatment center. This varies between hospitals and clinicians, but typically includes the provision of generic, written psychosocial support information from nursing and medical staff. Participants in the usual care condition did not have access to the Web-based information tool (which was password protected) or the telephone number to contact the study nurse. Contacts made by usual care participants with their health care team or access to other avenues of support were not monitored as part of the study.

\section{Data collection}

Outcome data were collected from participants using self-completed penand-paper surveys at 2, 4, 8, and 12 weeks post-recruitment. These time points were chosen as the intervention primarily targets the provision of care at diagnosis and during treatment, and it is during this time that patients are making decisions about, and receiving, intensive treatment. Baseline data were not collected as participants would most often have been provided with their diagnosis at the same time as being recruited into the study and completing a survey at this time was considered burdensome. SMS prompts, phone calls, and reminder letters including a second copy of the survey were used to encourage non-responders to complete and return the surveys.

\section{Outcome measures}

\section{Patients}

The primary outcome was the Health System and Information Needs Domain of the Supportive Care Needs Survey Short Form (SCNS-SF34). ${ }^{21}$ 
Participants indicated their level of need on a five-point scale: 1 "no need, not applicable"; 2 "no need, satisfied"; 3 "low need"; 4 "moderate need"; and 5 "high need." A domain score was calculated by summing item responses within the domain (minimum score 11, maximum 55), with a higher score indicating a higher level of unmet need. Anxiety and depression were secondary outcomes. Patients completed the Hospital and Anxiety Depression Scale (HADS), ${ }^{22}$ a 14 -item self-report instrument measuring anxiety and depression within the last seven days. Participants responded on a four-point scale, with 0 indicating the symptom is absent or almost absent, and 3 indicating that the symptom is severe or frequent. Scores from items were summed to provide an overall score out of 21 for each of the anxiety and depression subscales. Scoring was not conducted in real-time, therefore detection and treatment of high levels of anxiety and depression was the responsibility of their health care team as per usual care. For both SCNS and HADS, missing items were imputed as the mean of the individual's non-missing responses (within the subscale/domain) if only one item within each subscale/domain was missing.

\section{SPS}

The primary outcome for SPs was unmet needs assessed using the Information and Relationships Domain of the Support Persons Unmet Needs Survey. ${ }^{23}$ Participants provided responses on a five-point Likert scale from 0 (no unmet need) to 4 (very high unmet need). Domain scores were calculated, where at least one question answered in that domain, by summing item responses and dividing by the number of non-missing responses within the domain. A higher score indicates a higher level of unmet need. Secondary outcomes were measured using the Depression, Anxiety, Stress Scale-21 (DASS-21), ${ }^{23,24}$ a 21 item self-report instrument measuring depression, anxiety, and stress. Participants responded using a four-point scale from 0 (did not apply to me at all) to 3 (applied to me very much or most of the time). Scores for depression and anxiety were calculated by summing item responses within the scale and multiplying totals by two. Higher scores indicate greater prevalence and severity of symptoms. Scores were calculated if a patient had less than two items missing within each scale

\section{Use of the intervention}

Data regarding the mean number of times the website was accessed, the average length of each visit, and the content viewed, were captured by the software for each participant. Numbers of calls to the nurse support telephone number were documented. 


\section{Statistical analysis}

Originally, the study was powered assuming a sample of 340 patients and 280 SPs would be available at baseline. ${ }^{19}$ Assuming a $15 \%$ attrition at each followup, this would enable detection of differences between groups of 0.45 standard deviations (SDs) for patients and 0.5 SDs for SPs at 12 weeks follow up with $90 \%$ power and $5 \%$ significance for unmet needs. Due to difficulties recruiting participants, the total number recruited was much lower, providing $80 \%$ power to detect a $0.75 \mathrm{SD}$ difference for patient outcomes between groups at 12 weeks (assuming a correlation of 0.6 for repeated measures).

All individuals involved in analysis were blind to condition allocation until analysis was complete. Analysis followed the intention to treat principle. Due to the small sample size, only descriptive statistics for unmet needs, depression, and anxiety are presented for SPs. Patient outcomes were compared between the intervention and usual care groups at each time point using a mixed effects linear regression including data from all time points with fixed effects for group, time and interaction between time and group and clinic, and a random person-level intercept. Treatment group comparisons were estimated by least-square means for the group and are presented as a mean with $95 \%$ confidence intervals. $p$-Values for the within-group changes over time were also assessed for statistical significance. Primary analysis involved complete case analysis, and sensitivity analyses were conducted via multiple imputation where 10 datasets were imputed using the fully conditional specification (FCS) method. Predictive mean matching was used to impute the continuous variables: the outcomes of interest for all time-points; time since diagnosis; and average travel time. Categorical variables (gender, age, marital status, Aboriginal or Torres Strait Islander origin, education, and diagnosis) were imputed using the discriminant function. Results from the mixed models on each imputed dataset were combined using Rubin's rules.

The most significant biological factor determining clinical outcome in high grade hematological malignancy is the attainment of a complete remission (CR). Assessment of CR status occurs approximately 4 weeks after commencement of initial chemotherapy. Post hoc analyses of trends over time in information needs, anxiety, and depression were conducted to explore differences between participants who achieved remission and those who did not using linear mixed models. Statistical analyses were programed using SAS software v9.4. ${ }^{25}$

\section{Results}

\section{Participants}

A total of 81 patients were recruited. Four patients were subsequently excluded due to a histopathological diagnosis which did not meet eligibility 
criteria. This meant 38 patients were randomized to the intervention and 39 patients were randomized to usual care. At the first data collection time point, eight patients had withdrawn or died and nine were too unwell or did not respond. This provided 30 patients in the intervention group and 30 patients in the usual care group for analysis. The demographics of included patients and SPs are provided in Tables 1 and 2. Fifteen SPs were recruited, with the majority the spouse of a participant $(n=10)$.

\section{Comparison of unmet information needs for patients}

Patient unmet information needs scores are presented in Figure 1(A), and adjusted mean differences are presented in Table 3. There were no statistically significant differences in unmet information needs between groups at any time-point. Sensitivity analysis using multiply imputed data indicated the same trends (results not shown). Patients in both groups reported a decrease in unmet information needs from 2 to 12 weeks following diagnosis. In the usual care group, mean scores on the health systems and information domain started at $29.5(95 \%$ CI 26.2, 32.8) at week 2, then significantly decreased at each time point $(p=<0.001)$. In the intervention group, mean scores on the health systems and information domain at week 2 were 25.3 (95\% CI 22.0, 28.6), increased at week 4 , decreased to week 8 , and increased slightly to week $12(p=<0.007)$.

\section{Comparison of anxiety and depression for patients}

Patient anxiety and depression scores are presented in Figure 1(B,C) (and between group differences in Table 3). For the complete case analysis, a statistically significant difference in anxiety was observed at week 2 with the usual care group 2.34 points higher than the intervention group (95\% CI $0.45,4.23) ; p=0.0155$. There were no further statistically significant differences in anxiety or depression scores between groups. Results from the sensitivity analysis were consistent with the complete case analyses (results not shown).

\section{Comparison of unmet needs, depression, and anxiety according to disease remission status (post hoc analysis)}

Patient unmet information needs, anxiety, and depression scores by remission status are presented in Figure 2. Eight patients did not obtain remission. These patients were older $(p=0.049)$ and predominantly diagnosed with AML. The difference between those who did and did not achieve remission observed mean SCNS-SF34 scores over time was significant at the $10 \%$ but not $5 \%$ level $(p=0.084)$. The change in mean 
Table 1. Characteristics of patients completing the week 2 survey $(n=60) .^{\text {a }}$

\begin{tabular}{llcr}
\hline Variable & \multicolumn{1}{c}{ Category } & Control $(n=30)$ & Intervention $(n=30)$ \\
\hline Age (years) & Mean (SD) & $49(16)$ & $51(15)$ \\
Time since diagnosis (days, cont) & Mean (SD) & $25(15)$ & $23(12)$ \\
Gender & Male & $24(80 \%)$ & $17(57 \%)$ \\
Marital status & Female & $6(20 \%)$ & $13(43 \%)$ \\
& Single & $4(13 \%)$ & $5(17 \%)$ \\
Highest level of education & Married or de facto & $26(87 \%)$ & $21(70 \%)$ \\
& Divorced or widowed & $0(0 \%)$ & $4(13 \%)$ \\
Aboriginal or Torres Strait Islander Origin & Primary or secondary & $12(40 \%)$ & $5(17 \%)$ \\
& Trade & $8(27 \%)$ & $10(33 \%)$ \\
Diagnosis & Yniversity & $10(33 \%)$ & $15(50 \%)$ \\
& No & $2(6.7 \%)$ & $0(0 \%)$ \\
& Acute myeloid & $28(93 \%)$ & $30(100 \%)$ \\
& Acute lymphoblastic & $7(55 \%)$ & $19(66 \%)$ \\
Time since diagnosis & Burktitt Lymphoma & $4(14 \%)$ & $8(28 \%)$ \\
Average time to travel to cancer center & Lymphoblastic & $2(6.9 \%)$ & $1(3.4 \%)$ \\
& 2 weeks or less & $2(6.9 \%)$ & $1(3.4 \%)$ \\
& Over 2 weeks & $27(93 \%)$ & $3(12 \%)$ \\
& Ovr or less & $24(96 \%)$ & $23(88 \%)$ \\
& Over 1 hr & $1(4.0 \%)$ & $20(80 \%)$ \\
\hline
\end{tabular}

${ }^{\mathrm{a}}$ Note. Totals may not add to 30 per group due to missing data.

SCNS-SF34 scores across time for patients who did not achieve remission was marginally non-significant at the $5 \%$ level $(p=0.06)$; however, there was a significant decrease across time from 27.92 (95\% CI 25.26, 30.59) at week 2 to $24.29(95 \%$ CI $21.77,26.80)$ at week $12(p=<0.001)$ for patients who did achieve remission.

HADS anxiety scores did not differ significantly over time for patients who achieved remission $(p=0.266)$. For patients who did not achieve remission, mean anxiety scores significantly increased over time from 8.76 $(95 \%$ CI $5.87,11.65)$ at week 2 to $10.84(95 \%$ CI $8.88,12.81)$ at week 12 $(p=0.008)$. There was a significant difference in anxiety trajectories over time, with mean anxiety higher and increasing over time for those who did not achieve remission compared to declining scores in the remission group $(p=0.003)$. HADS depression scores did not change over time for those who achieved remission $(p=0.659)$ and those that did not $(p=0.878)$, and there was no significant interaction of group with time $(p=0.938)$.

\section{Unmet needs, depression and anxiety for SPS}

SPs unmet information needs, depression, and anxiety mean scores by intervention group are presented in Table 4. SPs in both groups reported low unmet needs at all time points on the SPUNS Information and Relationships domain. SPs in the control group scored normal to mild levels of depression, decreasing over time, while for SPs in the intervention group, depression scores increased from mild to moderate over time. Anxiety scores were in the normal-mild range at all time points for SPs in 
Table 2. Characteristics of SPs completing the week 2 survey $(n=15)$.

\begin{tabular}{llcc}
\hline Variable & \multicolumn{1}{c}{ Category } & Control $(n=8)$ & Intervention $(n=7)$ \\
\hline Age & Mean (SD) & $54(10)$ & $52(12)$ \\
Gender & Male & $4(50 \%)$ & $0(0 \%)$ \\
Marital status & Female & $4(50 \%)$ & $7(100 \%)$ \\
& Married or de facto & $7(88 \%)$ & $6(86 \%)$ \\
Highest level of education & Separated & $0(0 \%)$ & $1(14 \%)$ \\
& Divorced or widowed & $1(13 \%)$ & $0(0 \%)$ \\
Aboriginal or Torres Strait Islander Origin & Primary or secondary & $2(25 \%)$ & $1(14 \%)$ \\
Employment & Trade & $3(38 \%)$ & $2(29 \%)$ \\
& University & $3(38 \%)$ & $4(57 \%)$ \\
& No & $8(100 \%)$ & $7(100 \%)$ \\
& Yes & $0(0 \%)$ & $0(0 \%)$ \\
& Full time & $3(38 \%)$ & $0(0 \%)$ \\
& Part time & $1(13 \%)$ & $4(57 \%)$ \\
Relationship to patient & Home duties & $1(13 \%)$ & $2(29 \%)$ \\
& Unemployed & $1(13 \%)$ & $0(0 \%)$ \\
& Retired & $1(13 \%)$ & $1(14 \%)$ \\
& Other & $1(13 \%)$ & $0(0 \%)$ \\
& Spouse & $5(63 \%)$ & $5(71 \%)$ \\
& Child or grandchild & $2(25 \%)$ & $0(0 \%)$ \\
& Parent & $0(0 \%)$ & $1(14 \%)$ \\
& Sibling & $0(0 \%)$ & $1(14 \%)$ \\
& Other & $1(13 \%)$ & $0(0 \%)$ \\
\hline
\end{tabular}

both groups, except for an increase at week 4 for SPs in the control group, and an increase at week 12 for SPs in the intervention group.

\section{Use of the intervention}

All intervention patients accessed the website at least once. On average, patients accessed the website three times (minimum 1, maximum 10; Figure $3(\mathrm{~A})$ ), watched 3.6 videos (minimum 0 , maximum 9) and visited 4.3 pages (minimum 0, maximum 7). The average length of each visit was $31 \mathrm{~min}$ (minimum 1, maximum $134 \mathrm{~min}$ ). Information about blood cancer, its causes and treatments options were the most visited pages (Figure 3(B,C)). While patterns of access varied between patients, $79 \%$ of patients did not access the website again after the first 14 days post-recruitment. It was not possible to discern SP participants who used the website from other individuals with whom the patient may have shared the website, such as other family members or health care providers. The website was accessed on average 2.2 times (minimum 1, maximum 7) by non-patient users with a mean length of each visit of $25 \mathrm{~min}$ (minimum 2, maximum $122 \mathrm{~min}$ ). Non-patient users watched an average of 3.9 videos (minimum 0 , maximum 7) and visited 5.2 web pages (minimum 1, maximum 7). No study participants contacted the nursedelivered telephone support service or posted in the discussion forum.

\section{Discussion}

This trial examined the potential benefit of an online information tool and telephone support for individuals diagnosed with a high grade hematological 


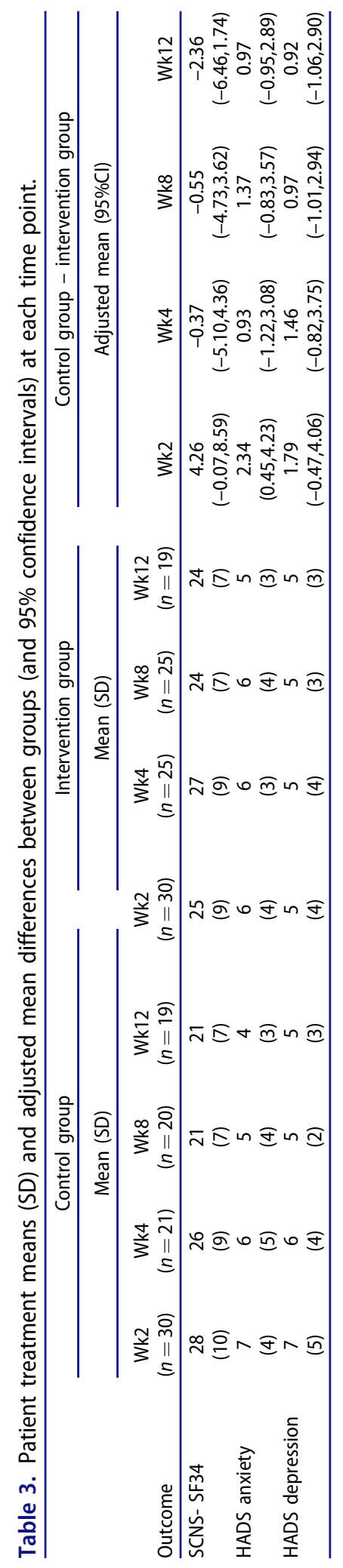



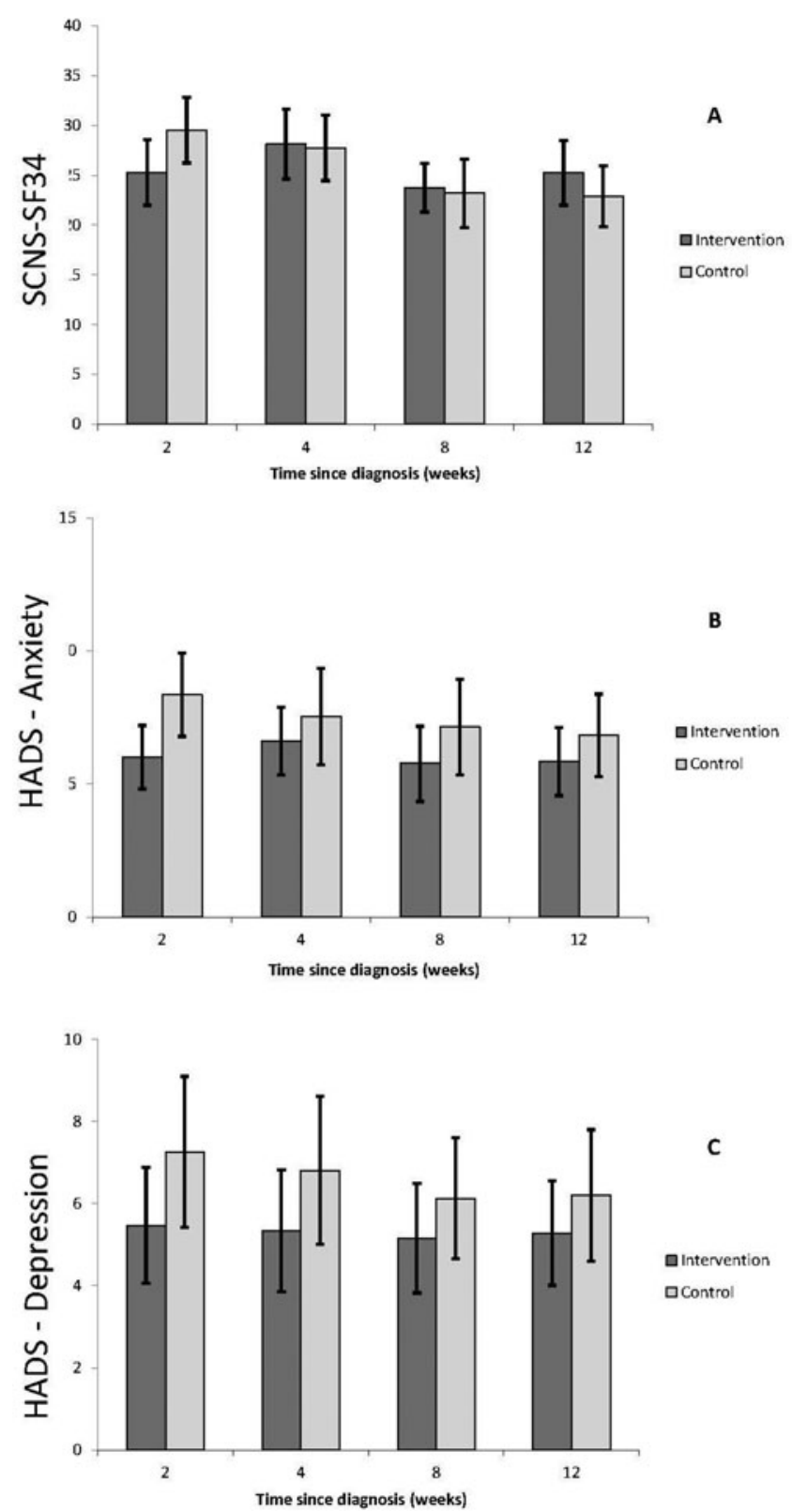

Figure 1. Patient scores (complete case analysis) at 2, 4, 8 and 12 weeks after diagnosis for: (A) unmet information needs (SCNS-SF34); (B) anxiety (HADS); and (C) depression (HADS). Data are presented for each group as a least-square mean with $95 \%$ confidence intervals.

malignancy. It differs from recent published trials examining the effectiveness of online interventions, as it focused on inpatients, and randomization occurred early in the disease course, prior to initiation of treatment. ${ }^{26-28}$

This trial demonstrates an early intervention approach to meeting the psychosocial needs of hematological cancer patients and their SPs is feasible but logistically difficult, which may have contributed to the inability to 

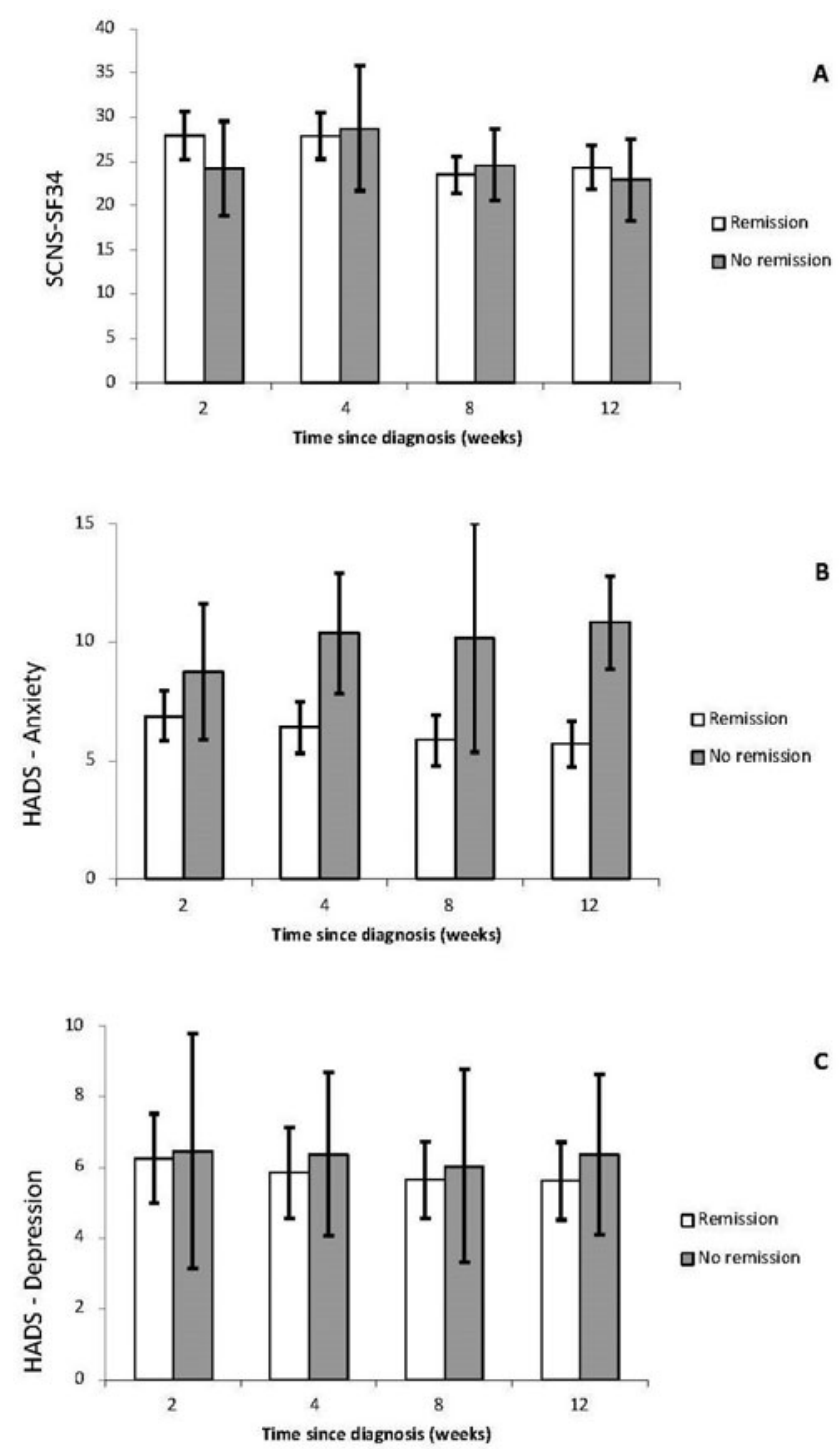

Figure 2. Patient scores (complete case analysis) at 2, 4, 8 and 12 weeks after diagnosis by readmission status for: (A) unmet information needs (SCNS-SF34); (B) anxiety (HADS); and (C) depression (HADS). Data are presented for each group as a least-square mean with $95 \%$ confidence intervals.

recruit the pre-defined target sample size. ${ }^{19}$ Patients with high grade hematological malignancy are typically admitted to hospital acutely unwell, receive their diagnosis quickly, begin treatment within a few days and then remain in hospital for $4-5$ weeks. This protocol required consent for trial participation and access to the intervention to be provided within the short time period prior to chemotherapy commencement, which contributed to the difficulty in achieving the required sample size. While we hypothesized that provision of the online tool would be beneficial during this time when 


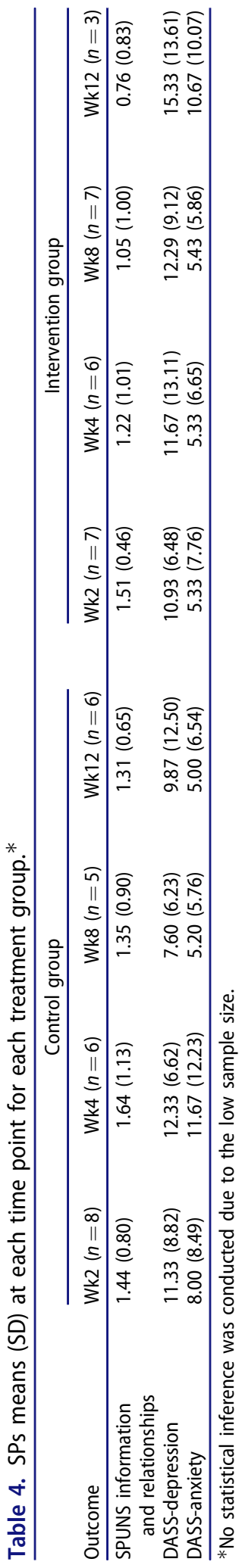



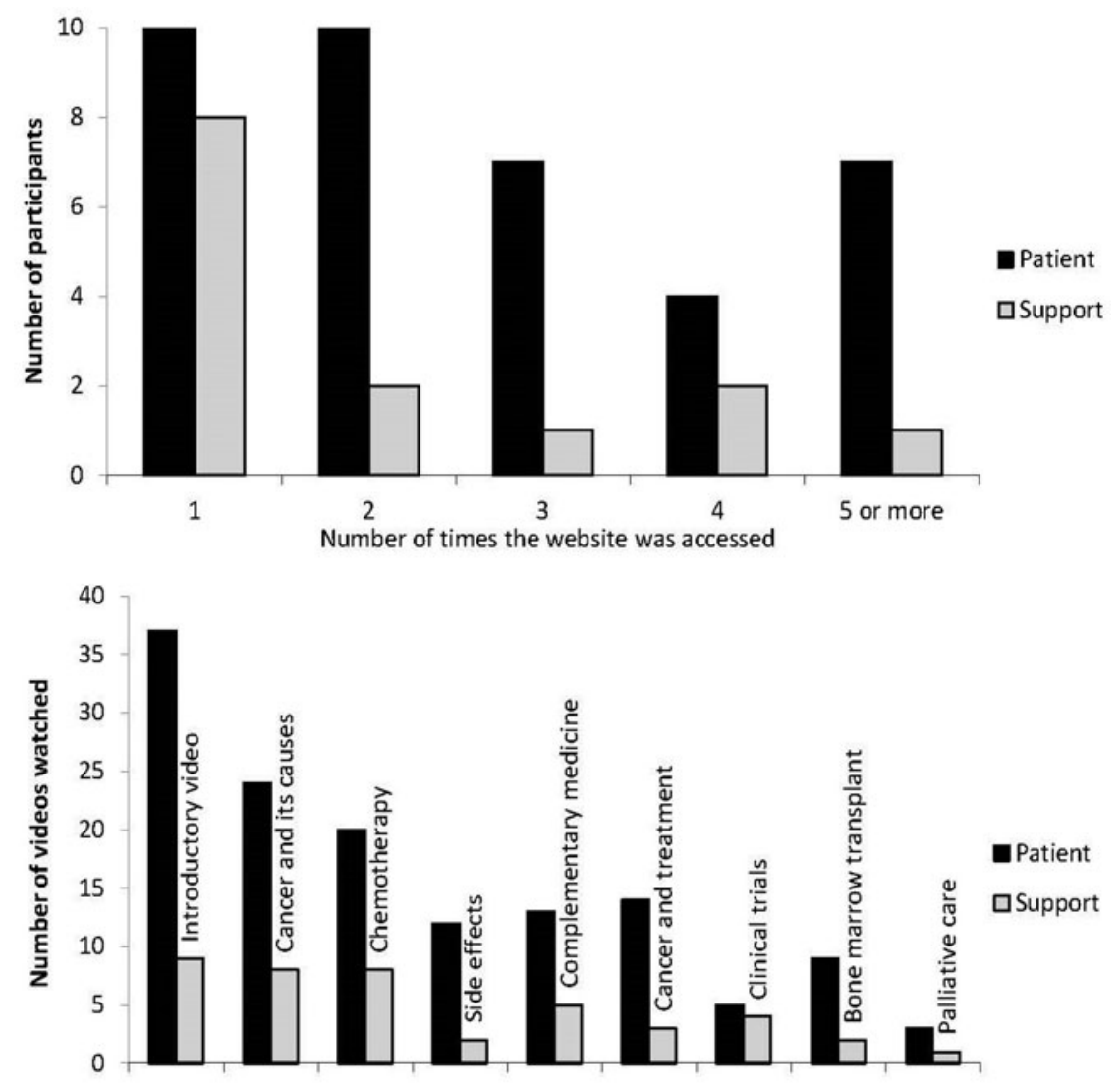

Information domains of website videos

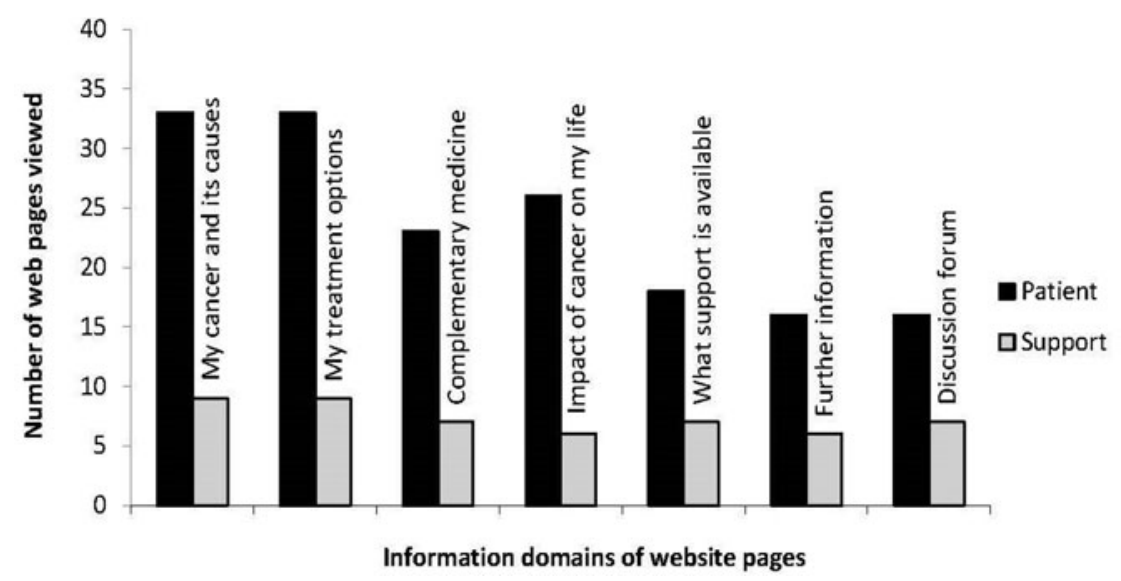

Figure 3. Use of the Web-based intervention. (A) Number of times the website was accessed by patient and non-patient users. (B) The frequency of visits to specific content areas in video format. (C) The frequency of visits to specific content areas in written format. 
participants were undergoing painful procedures, we recognize patients also received significant amounts of medical information from medical and nursing staff, rendering the tool potentially less effective through replication of information from other sources. This overlap of information provision was not anticipated prior to selecting the patient sample; rather the webbased tool was expected to reduce the complexity and burden for health care providers associated with meeting patients' psychosocial needs. Although not undertaken as part of this study, future evaluation of webbased tools should consider utility and impact of the tool from the perspective of the health care provider. Immediate access to information from health care providers due to the nature of inpatient care may also explain the poor uptake of the nurse-delivered telephone support service. Such supports may have more utility for an outpatient sample.

No statistically significant effect on unmet information needs for patients was found. This may, in part, be the result of both inadequate sample size to detect a difference, and informal pathways of information provision to the usual care group throughout the study. Importantly, patients in both intervention and usual care groups experienced significant reductions in unmet needs over the data collection time periods. It would be useful to examine the effectiveness of a Web-based information tool for patients with blood cancers that are treated on an outpatient basis (e.g. Diffuse large B-cell lymphoma or Hodgkin Lymphoma). Web-based information tools may have greater benefits for newly diagnosed outpatients, given they are likely to receive less information transfer from staff due to time-limited interactions and have a greater decision-making burden. The spectrum of therapies offered for some low-grade lymphomas treated in the outpatient setting vary from multi-agent chemotherapy to observation only. Future research in this area should consider undertaking a comprehensive needs assessment with the target audience as part of the development process to ensure utility and value-adding of the information included in the webbased tool beyond that which is already available via usual care.

Unmet information needs have been identified as the most significant domain of unmet needs for acute leukemia patients. ${ }^{29}$ A cohort of 311 leukemia patients from China $^{29}$ administered the SCNS-SF34 identified a mean health information score of 51. In this study, the mean scores in the health information needs domain in the usual care and intervention arms at week 2 were 29.5 and 25.3, respectively, and then scores fell over time. These measurements of unmet need, obtained approximately 2 to 3 weeks after initial blood cancer diagnosis, were substantially less than the Chinese leukemia cohort and were not very different to a mixed sample of 1516 cancer survivors (mean score 23.9), with predominantly breast, prostate, colorectal and lung cancer from Connecticut in the USA. ${ }^{30}$ The relatively 
low health information scores identified in this cohort at the first time point may reflect patients obtaining health information during prolonged hospital admission. Access to health information may reduce as health care is transitioned into the outpatient treatment setting and subsequently the survivor population following treatment.

Post hoc analyses revealed individuals who did not obtain remission with initial treatment had a significant increase in anxiety. This subgroup of patients is at increased risk of psychological distress, and interventions aimed at reducing anxiety may be most effectively targeted at this group.

\section{Limitations}

Results should be considered in light of several limitations. First, recruiting patients before they commenced treatment was difficult due to the acuity of illness, and the speed at which patients started treatment. This meant the prespecified sample size was not achieved. Therefore, the study had limited power to detect differences in outcomes. Additionally, the nurse delivered telephone support line was not accessed by any participants in the intervention. Thus, the effectiveness of this component was not tested as intended.

\section{Conclusion}

A Web-based information tool and nurse delivered telephone support did not significantly reduce unmet information needs, depression, or anxiety among hematological cancer patients; however, this finding is inconclusive given the low power of the study.

\section{Author contributions}

All authors were involved in design of the clinical trial. RSF, WS, AW, FH, MC, CDE, and CP obtained study funding. WS, JB, RW, RSF, and AW developed the intervention. WS, JB, RW, and RSF oversaw overall implementation of the trial. WS, CB, SR, CT, AE, and JG oversaw implementation of the trial at specific sites. FH developed the underlying software for the web-based tool. CO conducted data analysis. All authors have contributed to, read, and approved the final manuscript.

\section{Compliance with ethical standards}

Conflict of Interest: The authors declare that they have no conflict of interest.

Ethical Approval: All procedures performed in studies involving human participants were in accordance with the ethical standards of the institutional and/or national research committee and with the 1964 Helsinki declaration and its later amendments or comparable ethical standards. Approval was also provided by the local research governance committees of each participating hospital. 
Informed Consent: Informed consent was obtained from all individual participants included in the study.

\section{Acknowledgements}

The authors would like to thank the members of the Expert Advisory Groups for assistance with development of the intervention, participating treatment centers for assisting with recruitment of patients, our volunteers who assisted with intervention implementation and data collection, Dr Breanne Hobden for assistance with trial management and data collection, and Dr David Paul for assistance with development of the web-based tool. We would like to thank Dr Andrew Wei and Associate Professor Mariko Carey for their advice with study design and intervention development. We would also like to thank the Cancer Council NSW, Leukemia Foundation, and MacMillan Cancer Support UK for permission to use and adapt information resources.

\section{Funding}

This trial was funded by a Cancer Institute New South Wales Translation Program Grant (10/THS/2-14). Dr Jamie Bryant was supported by an Australian Research Council PostDoctoral Industry Fellowship. Dr Anoop K Enjeti is the recipient of a Hunter New England Local Health District/NSW Health Pathology/Calvary Mater Newcastle Translational Research Fellowship. Dr Flora Tzelepis was supported by a Leukemia Foundation of Australia and Cure Cancer Australia Foundation Post-Doctoral Research Fellowship. Prof Christine Paul was supported by a National Health and Medical Research Council Career Development Fellowship (APP1061335).

\section{ORCID}

William Stevenson (D) https://orcid.org/0000-0002-0836-0313

Jamie Bryant (D) https://orcid.org/0000-0001-9378-5852

Rochelle Watson (D) https://orcid.org/0000-0003-3084-0417

Rob Sanson-Fisher (D) https://orcid.org/0000-0001-6022-2949

Christopher Oldmeadow (D) https://orcid.org/0000-0001-6104-1322

Frans Henskens (D) https://orcid.org/0000-0003-2358-5630

Christina Brown (D) https://orcid.org/0000-0003-2811-8943

Sundra Ramanathan (D) https://orcid.org/0000-0002-2744-2314

Campbell Tiley (D) https://orcid.org/0000-0002-4314-0877

Anoop Enjeti (D) https://orcid.org/0000-0001-8069-090X

Johanna Guest (D) https://orcid.org/0000-0002-9246-9054

Flora Tzelepis (D) https://orcid.org/0000-0002-9914-2732

Christine Paul (D) https://orcid.org/0000-0002-0504-5246

Catherine D'Este (D) https://orcid.org/0000-0001-9125-0483

\section{References}

1. McGrath P. Qualitative findings on the experience of end-of-life care for hematological malignancies. Am J Hosp Palliat Care. 2002;19(2):103-111. doi:10.1177/ 104990910201900208 
2. eviQ Cancer Treatments Online. https://www.eviq.org.au/. Accessed February 07, 2018.

3. Linden W, Vodermaier A, MacKenzie R, Greig D. Anxiety and depression after cancer diagnosis: prevalence rates by cancer type, gender, and age. J Affect Disord. 2012; 141(2-3):343-351. doi:10.1016/j.jad.2012.03.025

4. Molassiotis A, Wilson B, Blair S, Howe T, Cavet J. Unmet supportive care needs, psychological well-being and quality of life in patients living with multiple myeloma and their partners. Psychooncology. 2011;20(1):88-97. doi:10.1002/pon.1710

5. Stacey D, Bennett CL, Barry MJ, et al. Decision aids for people facing health treatment or screening decisions. Cochrane Database Syst Rev. 2011;1:CD001431.

6. Kinnersley P, Edwards AG, Hood K, et al. Interventions before consultations for helping patients address their information needs. Cochrane Libr. 2007;3:CD004565.

7. Holmes-Rovner M, Nelson WL, Pignone M, et al. Are patient decision aids the best way to improve clinical decision making? Report of the IPDAS Symposium. Med Decis Making. 2007;27(5):599-608. doi:10.1177/0272989X07307272

8. Watson PW, McKinstry B. A systematic review of interventions to improve recall of medical advice in healthcare consultations. J R Soc Med. 2009;102(6):235-243. doi:10. 1258/jrsm.2009.090013

9. Waller A, Forshaw K, Bryant J, et al. Interventions for preparing patients for chemotherapy and radiotherapy: a systematic review. Support Care Cancer. 2014;22(8): 2297-2308. doi:10.1007/s00520-014-2303-3

10. Leydon GM, Boulton M, Moynihan C, et al. Cancer patients' information needs and information seeking behaviour: in depth interview study. Br Med J. 2000;320(7239): 909-913. : doi:10.1136/bmj.320.7239.909

11. Cox A, Jenkins V, Catt S, Langridge C, Fallowfield L. Information needs and experiences: an audit of UK cancer patients. Eur J Oncol Nurs. 2006;10(4):263-272. doi:10. 1016/j.ejon.2005.10.007

12. National Breast Cancer and National Cancer Control Initiative. Clinical Practice Guidelines for Psychosocial Care of Adults with Cancer. Camperdown, NSW: National Breast Cancer Centre; 2003.

13. McPherson CJ, Higginson IJ, Hearn J. Effective methods of giving information in cancer: a systematic literature review of randomized controlled trials. J Public Health. 2001;23(3):227-234. doi:10.1093/pubmed/23.3.227

14. Mathieu E. The Internet and medical decision making: can it replace the role of health care providers?. Med Decis Making. 2010;30(5_suppl):14S-16S. doi:10.1177/ 0272989X10381228

15. Peterson MW, Fretz PC. Patient use of the internet for information in a lung cancer clinic. Chest. 2003;123(2):452-457. doi:10.1378/chest.123.2.452

16. Smits R, Bryant J, Sanson-Fisher R, et al. Tailored and integrated web-based tools for improving psychosocial outcomes of cancer patients: the DoTTI development framework. J Med Internet Res. 2014;16(3):e76. doi:10.2196/jmir.2849

17. Murray E, Burns J, See TS, Lai R, Nazareth I. Interactive Health Communication Applications for people with chronic disease. Cochrane Database Syst Rev. 2005;4: CD004274.

18. Nguyen HQ, Carrieri-Kohlman V, Rankin SH, Slaughter R, Stulbarg MS. Internetbased patient education and support interventions: a review of evaluation studies and directions for future research. Comput Biol Med. 2004;34(2):95-112. doi:10.1016/ S0010-4825(03)00046-5 
19. Bryant J, Sanson-Fisher R, Stevenson W, et al. Protocol of a multi-centre randomised controlled trial of a web-based information intervention with nurse-delivered telephone support for haematological cancer patients and their support persons. BMC Cancer. 2015;15(1):295.doi:10.1186/s12885-015-1314-x

20. Rodin G, Yuen D, Mischitelle A, et al. Traumatic stress in acute leukemia. Psychooncology. 2013;22(2):299-307.

21. Boyes A, Girgis A, Lecathelinais C. Brief assessment of adult cancer patients' perceived needs: development and validation of the 34-item Supportive Care Needs Survey (SCNS-SF34). J Eval Clin Pract. 2009;15(4):602-606. doi:10.1111/j.1365-2753. 2008.01057.x

22. Zigmond AS, Snaith RP. The hospital anxiety and depression scale. Acta Psychiatr Scand. 1983;67(6):361-370. doi:10.1111/j.1600-0447.1983.tb09716.x

23. Campbell SH, Sanson-Fisher R, Taylor-Brown J, Hayward L, Sunny Wang X, Turner D. The cancer support person's unmet needs survey: psychometric properties. Cancer. 2009;115(14):3351-3359. doi:10.1002/cncr.24386

24. Lovibond SH, Lovibond PF. Manual for the Depression Anxiety Stress Scales. 2nd ed. Sydney: Psychology Foundation; 1995.

25. SAS Institute. SAS v9.4 In. Cary, NC: SAS Institute; 2013.

26. Bouma G, de Hosson LD, van Woerkom CE, et al. Web-based information and support for patients with a newly diagnosed neuroendocrine tumor: a feasibility study. Support Care Cancer. 2017;25(7):2075. doi:10.1007/s00520-017-3598-7

27. Foster C, Grimmett C, May CM, et al. A web-based intervention (RESTORE) to support self-management of cancer-related fatigue following primary cancer treatment: a multi-centre proof of concept randomised controlled trial. Support Care Cancer. 2016;24(6):2445-2453. doi:10.1007/s00520-015-3044-7

28. Beatty L, Koczwara B, Wade T. Evaluating the efficacy of a self-guided Web-based CBT intervention for reducing cancer-distress: a randomised controlled trial. Support Care Cancer. 2016;24(3):1043-1051. doi:10.1007/s00520-015-2867-6

29. Yu FF, Bai YN, He H, et al. Identifying the unmet supportive care needs, with concomitant influencing factors, in adult acute leukemia patients in China. Eur J Oncol Nurs. 2017; 30:67-74. doi:10.1016/j.ejon.2017.07.003

30. Knobf MT, Ferrucci LM, Cartmel B, et al. Needs assessment of cancer survivors in Connecticut. J Cancer Surviv. 2012;6(1):1-10. doi:10.1007/s11764-011-0198-2 